Article

\title{
Impedance-Matched, Double-Zero Optical Metamaterials Based on Weakly Resonant Metal Oxide Nanowires
}

\author{
Diego R. Abujetas *, Ramón Paniagua-Domínguez ` and José A. Sánchez-Gil \\ Instituto de Estructura de la Materia (IEM-CSIC), Consejo Superior de Investigaciones Científicas, Serrano 121, \\ 28006 Madrid, Spain; ramon-paniag@dsi.a-star.edu.sg (R.P.-D.); j.sanchez@csic.es (J.A.S.-G.) \\ * Correspondence: diego.romero@iem.cfmac.csic.es; Tel.: +34-915616800 \\ + Current address: Data Storage Institute, A*STAR (Agency for Science, Technology and Research), \\ Singapore 138634, Singapore.
}

Received: 28 February 2018; Accepted: 22 March 2018; Published: 28 March 2018

\begin{abstract}
Artificial optical metamaterial with a zero index of refraction holds promise for many diverse phenomena and applications, which can be achieved with vacuum (or related) surface impedance and materials in the optical domain. Here, we propose simple metal-oxide nanorods as meta-atoms on the basis of an effective medium approach, based on their weak overlapping (electric/magnetic) resonances. We thus studied the optical properties of $\mathrm{TiO}_{2}$ nanowire arrays with a high-filling fraction through their photonic band structure, which exhibits a double-degeneracy point without a band gap at the center of the Brillouin zone. Various configurations are considered that reveal their performance over a reasonable range of incident wave vectors as impedance-matched, double-zero, bulk (low-loss) metamaterials.
\end{abstract}

Keywords: $\varepsilon$-near-zero metamaterials; double-zero metamaterials; metal-oxide nanowires

\section{Introduction}

In recent years, new phenomena have been observed in the field of metamaterials that would allow unprecedented control of the flow of electromagnetic waves, thus opening fascinating possibilities in different fields of physics and engineering. From the advent of negative-index metamaterials [1,2], other regimes of effective dielectric permittivity or magnetic permeability have been explored with tailored effective refractive indices, ranging from positive to negative values [3,4]. In particular, zero-index metamaterials [5-9] (effective refraction index near zero, $n \simeq 0$ ) have also become a subject of active investigation [10-17] due to their unique electromagnetic characteristics stemming from their vanishing $\varepsilon$, namely, the electromagnetic tunneling and energy squeezing that occurs through subwavelength narrow channels, which can be exploited in supercoupling [10,11], optical nanocircuit boards [18], waveguide coupling [19], directional [5,20] or position-independent emission enhancement [21], enhanced nonlinear effects [22,23], photonic doping [24], and even levitation [25]. Thus far, most investigated zero-index metamaterials are based on $\varepsilon$-near-zero (ENZ) metamaterials, although magnetic $\mu$-near-zero metamaterials would also yield $n \simeq 0$, exhibiting properties identical to those of $\varepsilon$-near-zero metamaterials $[26,27]$.

Attempts to fabricate zero-index metamaterials have been carried out in the microwave [14], $\mathrm{THz}$ [28], IR [23,29], and optical domains [17,30-32]. Nonetheless, like any other optical metamaterial, making them bulky and lossless is a major challenge [33-35]. Moreover, matching the impedance of metamaterials to that of a vacuum is crucial in many applications $[6,9,13,20,29,30]$. Therefore, the effective dielectric permittivity and magnetic permeability must reach zero simultaneously so that the resulting impedance-matched, double-zero metamaterial (DZM) exhibits $n \rightarrow 0$ and $Z=\sqrt{\mu / \varepsilon} \sim 1$. 
However, as is known, supercoupling effects, such as a perfect conductor slab or a waveguide filled with material with high $Z$ values (where both $\varepsilon$ and $\mu$ neither match nor approach zero with the same dispersion profile) can arise at specific configurations [9-11,27,36].

In this regard, one approach has relied on high-index all-dielectric photonic crystals $[15,30,31]$, exploiting the emergence of an accidental-degeneracy-induced Dirac point at the center of the Brillouin zone, which leads to an effective DZM behavior [15]. In this respect, it should be pointed out that (non-magnetic) high-index dielectric meta-atoms (such as semiconductor nanoparticles) can deliver effective optical magnetic responses through Mie-like magnetic resonances [37-51], which, combined with the well known negative electric response of metals in the visible range, might lead to bulky negative-index metamaterials [34,35,52,53] or cloaking devices [54-57].

In this work, we show theoretically that the combination of weak (electric and magnetic) resonances, slightly blue-shifted, in lossless dielectric nanoparticles suffice to achieve optical double-zero metamaterials in such a way that the corresponding impedance remains close to that of the vacuum. In particular, we propose dielectric nanorods with a lossless, moderately large dielectric function $\varepsilon$ (as is the case of metal oxides), made of materials that can potentially span the whole visible range, at the expense of high-filling fractions, as excellent candidates for the meta-atoms of such impedance-matched, optical DZMs, revealing numerically their remarkable performance as DZM supercoupling devices in two dimensions based on arrays of titanium oxide nanowires.

\section{Results}

\subsection{An Effective Medium Approach}

Let us first analyze through a simple theoretical model the requirements for a nearly-zero-dielectric permittivity and magnetic permeability. To that end, we have extended the effective medium theory of magnetodielectric composites developed specifically for cylinders or spheres, [58] which reduces in the quasi-static limit to the Maxwell-Garnett theory. In the (2D) case of infinitely long nanowires of radius $R$, with dielectric permittivity $\varepsilon_{\mathcal{C}}$ (non-magnetic $\mu_{c}=1$ ), embedded in vacuum $\left(\varepsilon_{0}, \mu_{0}\right)$ with filling fraction $f$, we obtain generalized expressions for the effective anisotropic $\tilde{\varepsilon}_{\text {eff }}$ and $\tilde{\mu}_{\text {eff }}\left(\varepsilon_{\|}^{\text {eff }}\right.$ and $\mu_{\|}^{\text {eff }}$ in the plane perpendicular to the nanowire axis and $\varepsilon_{z}^{\text {eff }}$ and $\mu_{z}^{\text {eff }}$ along the nanowire axis) in terms of analogue dipolar polarizibilities, as follows:

$$
\begin{aligned}
& \frac{\varepsilon_{\|}^{\text {eff }}-\varepsilon_{0}}{\varepsilon_{\|}^{\text {eff }}+\varepsilon_{0}}=-f \frac{\alpha_{\|}^{(E)}}{\pi R^{2}} \\
& \frac{\mu_{z}^{\text {eff }}}{\mu_{0}}-1=-f \frac{\alpha_{z}^{(H)}}{\pi R^{2}}
\end{aligned}
$$

for transverse electric (TE) waves, that is, the electric field linearly polarized perpendicular to the nanowire axis; note that, due to the symmetry of Maxwell's equations, the effective medium parameters for transverse magnetic (TM) waves can be obtained from those of TE waves by exchanging permittivities, permeabilities, and polarizibilities. These expressions allow us to roughly determine what type of (or spectral regime of) polarizabilities will be a priori suitable to yield specific effective medium behaviors. For instance, a negative refractive index ( $\left.\varepsilon_{\|}^{\text {eff }} \simeq-1, \mu_{z}^{\text {eff }} \simeq-1\right)$ requires from Equation (2) very high electric dipolar polarizibility $1 / \alpha_{\|}^{(E)} \rightarrow 0$, which is in turn the approach followed in $[34,35]$ through overlapping electric and magnetic Mie resonances in dense arrays of core-shell metal-semiconducting nanowires. One would expect that a similar approach would suffice to achieve DZMs with the advantage of not needing such large filling fractions. Though this is plausible, the on-resonance condition introduces moderately large (scattering) losses that may in turn kill the zero-index behavior through the imaginary parts of $\alpha_{\|}^{(E)}$ and $\alpha_{z}^{(H)}$. 
Nonetheless, another approach can be inferred from Equation (2) upon assuming that $\varepsilon_{\|}^{\text {eff }}$ and $\mu_{z}^{\text {eff }}=0$. The required dipolar polaribilities can be written as

$$
\begin{aligned}
\alpha_{\|}^{(E)} & \simeq f^{-1} \pi R^{2} \\
\alpha_{z}^{(H)} & \simeq f^{-1} \pi R^{2} .
\end{aligned}
$$

Such electromagnetic (EM) conditions can be fulfilled on the blue side of the spectral regime of weakly resonant scattering of dielectric nanorods. Since only moderately large dielectric constants are needed, metal oxides can be exploited with negligible losses in most of the optical domain [59], in turn synthesized as nanowires with suitable dimensions [60]; as e.g., $\mathrm{TiO}_{2}$ nanowires, which can be easily fabricated through thermal evaporation [61]. Upon plane wave illumination, the scattering and extinction cross sections (and thus the absorption) of cylinders can be expanded as sums over different multipolar contributions [34]. For a TE wave:

$$
\begin{array}{r}
Q_{s c a}=\frac{2}{k R}\left(\left|a_{0}\right|^{2}+2 \sum_{j=1}^{\infty}\left|a_{j}\right|^{2}\right) \\
Q_{e x t}=\frac{2}{k R} \operatorname{Re}\left(a_{0}+2 \sum_{j=1}^{\infty} a_{j}\right)
\end{array}
$$

where $k=2 \pi / \lambda, \lambda$ being the wavelength of the external medium. For dielectric particles $(n=\sqrt{\varepsilon})$, the coefficients $a_{j}$ are given by

$$
a_{j}=\frac{n J_{j}^{\prime}(k R) J_{j}(n k R)-J_{j}(k R) J_{j}^{\prime}(n k R)}{n J_{j}(n k R) H_{j}^{\prime}(k R)-H_{j}(k R) J_{j}^{\prime}(n k R)}
$$

where $J_{j}$ and $H_{j}$ are the standard Bessel and (first-kind) Hankel functions, and the primes indicate differentiation with respect to the argument. Each coefficient $a_{j}$ represents a certain multipolar character that can be either electric or magnetic. The first two lowest-order terms, $a_{0}$ and $a_{1}$, are related to the magnetic and electric dipole polarizabilities, respectively, while higher-order terms are related to other multipolar contributions. The Mie extinction (or scattering, since absorption is neglected thus far) cross section of an infinite $\mathrm{TiO}_{2}$ nanocylinder of radius $R=180 \mathrm{~nm}$ in TE polarization (the electric field perpendicular to the cylinder axis) is shown in Figure 1; incidentally, note that the polarization is the opposite to that in $[15,30,31]$. The refractive index of $\mathrm{TiO}_{2}$ is assumed constant $n_{\mathrm{TiO}_{2}}=2.6$ throughout the spectral region studied therein [59]. In addition, we show separately the lowest-order multipolar contributions to the Mie extinction cross section. The electric-dipole resonance is clearly observed, stemming from the $a_{1}$ term in Mie scattering, which in turn gives the contribution to the electric polarizibility $\alpha_{\|}^{(E)}$. On the other hand, the contribution from the $a_{0}$ term yields a very weak magnetic resonance in the infrared (not shown in Figure 1), providing the contribution to the (effective) magnetic polaribility $\alpha_{z}^{(H)}$. Both are negative in the overlapping regime in the blue part (at smaller wavelengths) of the electric/magnetic resonances, exhibiting appropriate DZM conditions (3) as explicitly indicated. Snapshots of the EM field components (in-plane electric and out-of-plane magnetic) for an incoming field propagating along the $x$ axis (from left to right) are shown at and off resonance. The field distributions illustrate their behavior opposing the incident fields on the blue side of the resonances $(\lambda=750 \mathrm{~nm})$. 

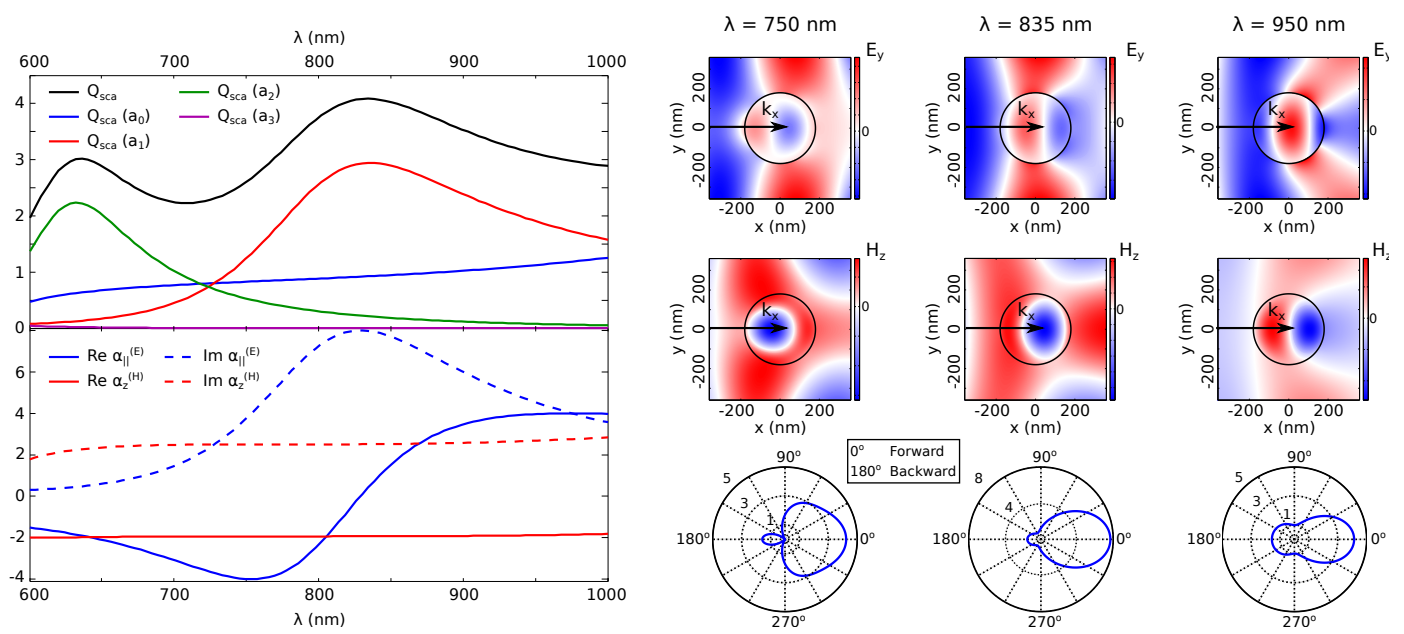

Figure 1. Left: (top) Mie extinction efficiency $Q_{\text {ext }} \equiv Q_{\text {sca }}$ in TE polarization and (bottom) corresponding polarizibilities $\alpha_{\|}^{(E)}$ and $\alpha_{z}^{(H)}$ for a $\mathrm{TiO}_{2}$ nanocylinder of radius $R=180 \mathrm{~nm}$. Right: (top) Contour maps of the transverse electric and longitudinal magnetic fields, and (bottom) angular scattering patterns, at the $a_{1}$ Mie resonance $(\lambda=835 \mathrm{~nm})$ and off resonance, at both higher and lower wavelengths ( $\lambda=950 \mathrm{~nm}$ and $\lambda=750 \mathrm{~nm}$, respectively). The incident light propagates from left to the right along the $x$ axis and the electric field lies on the $y$ axis.

Next, we exploit the effective medium (Equation (2)) to obtain the effective parameters expected for a random arrangement of $\mathrm{TiO}_{2}$ nanorods. This is shown in Figure 2 for varying filling fractions. It can be seen that, for increasing filling fraction $f$, the real parts of $\varepsilon_{\|}^{\text {eff }}$ and $\mu_{z}^{\text {eff }}$ tend to decrease; this is true not only for their real parts but also their imaginary parts, which account for the effective absorption. These, however, remain reasonably low. Since the material is lossless, effective absorption losses stem only from scattering losses. In this regard, we have plotted the scattering pattern associated to single Mie nanocylinders at several frequencies in Figure 1. Note that, though the scattering cross section is moderately large in the blue part of the electric resonance (spectral regime appropriate for DZM behavior), the angular pattern reveals strong suppression of backward scattering, which might contribute to the minimization of scattering losses in the effective medium. With the help of the above (effective medium) parameters, we expect the resulting effective refractive index $n^{\text {eff }}=\sqrt{\varepsilon_{\|}^{\text {eff }} \mu_{z}^{\text {eff }}}$ to approach zero with a moderate impedance.
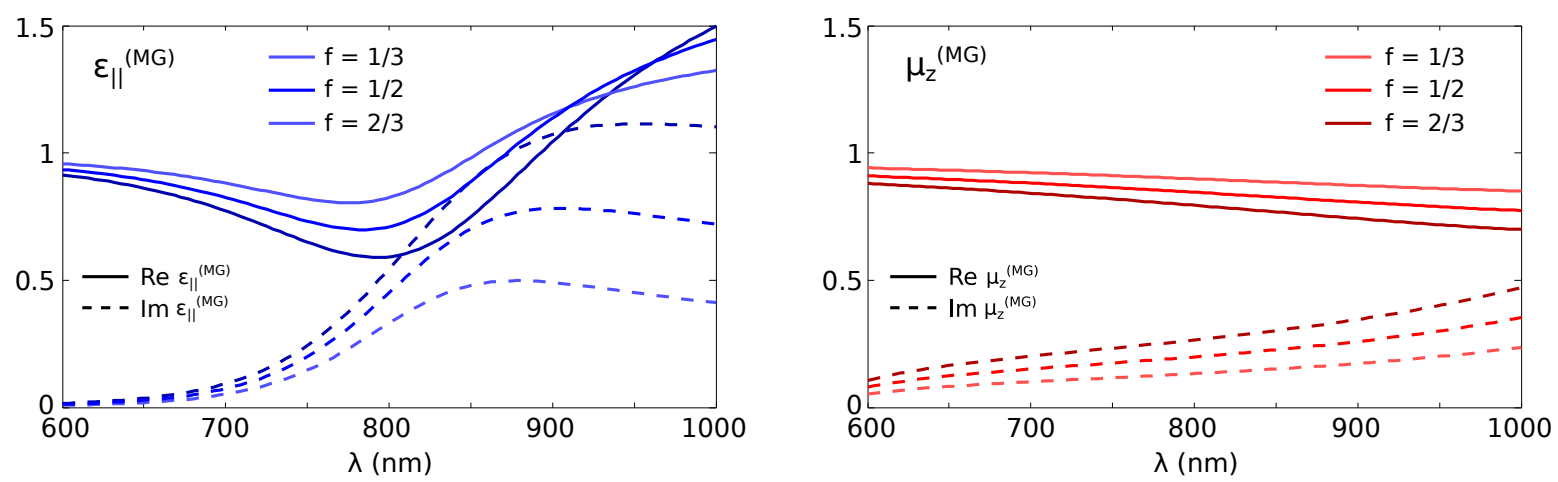

Figure 2. Spectral dependence of the effective parameters (left) $\varepsilon_{\|}^{\text {eff }}$ and (right) $\mu_{z}^{\text {eff }}$ in TE polarization for a random arrangement of $\mathrm{TiO}_{2}$ nanocylinders of radius $R=180 \mathrm{~nm}$ with varying filling fractions $f=1 / 3,1 / 2,2 / 3$. 


\subsection{Numerical Calculations: Double-Zero Metamaterials}

Since the required filling fractions $f \geq 2 / 3$ exceed the limit of validity of the effective medium theory, let us explore numerically the EM behavior of a metamaterial consisting of such $\mathrm{TiO}_{2}$ nanorods. To that end, a triangular lattice of $\mathrm{TiO}_{2}$ is considered with a period $(d=380 \mathrm{~nm})$, such that the resulting filling fraction $f \sim 0.8$ belongs to the spectral regime of interest $n^{\text {eff }} \sim 0$, predicted above. First of all, we show in Figure 3 the resulting photonic band structure. The region of interest lies near the $\Gamma$ point at frequencies above the pseudo-gap of the second band, where the third and fourth bands appear degenerate. Incidentally, this spectral region roughly coincides with that of the electric dipole, the $a_{1}$ Mie resonance mentioned in the previous subsection. This region has been magnified for the sake of clarity in Figure 3: A double degeneracy is observed at the $\Gamma$ point with nearly isotropic quadratic bands with increasing $k$. It should be emphasized that no (accidental) triply-degenerate Dirac point is achieved as mentioned in $[15,30,31,62]$ for opposite linear polarization, different lattice symmetry, and higher refractive index contrast. However, as mentioned in [63], if indeed our system can be made homogeneous, the zero phase velocity with the high group velocity associated with the obtained bands could lead to DZM behavior near the $\Gamma$ point. It should be recalled that, if the effective index of refraction is actually close to zero, the effective wavelength inside could be large enough to consider the medium homogenizable [15].

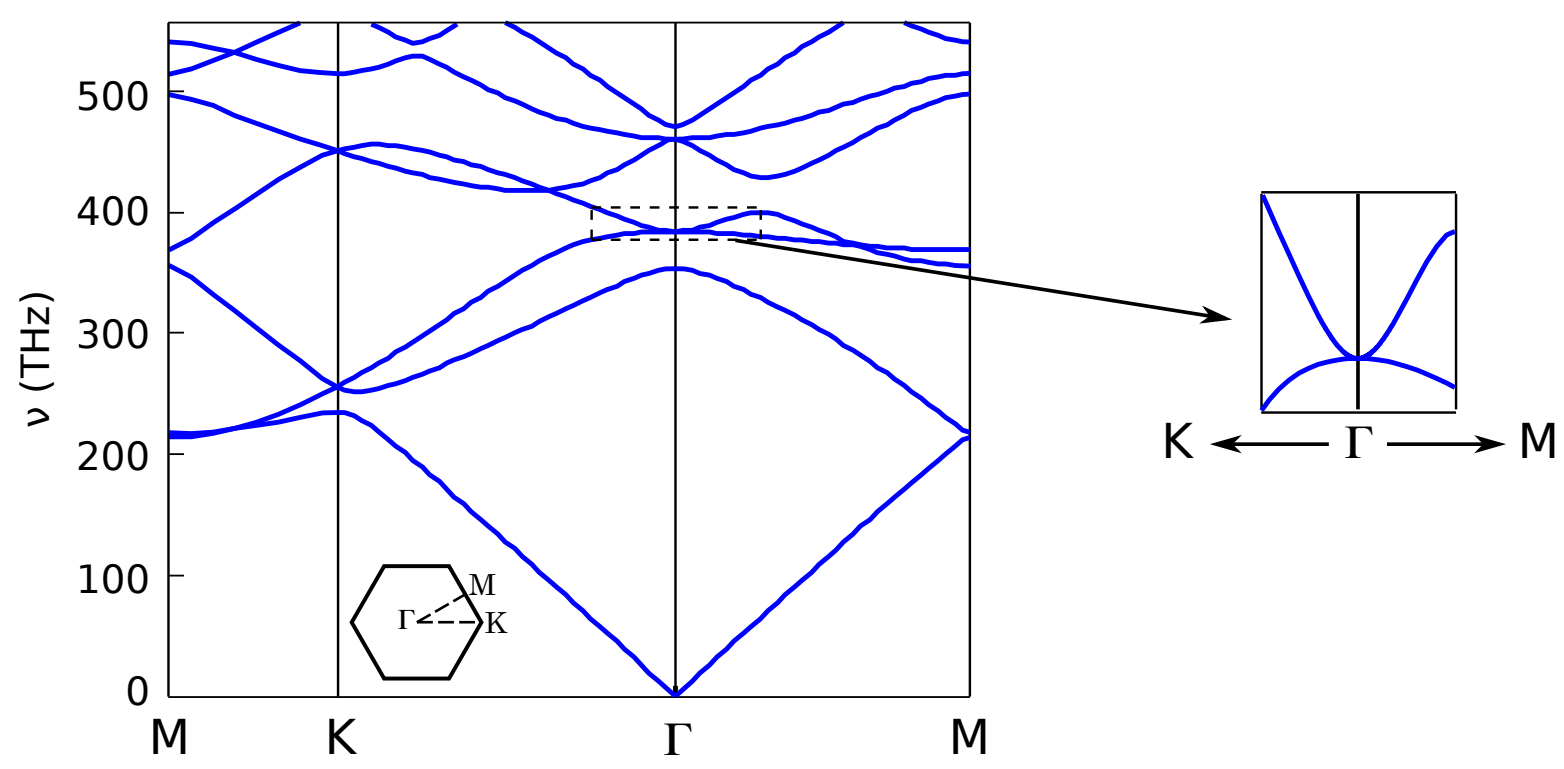

Figure 3. 2D photonic band structure for a triangular lattice of $\mathrm{TiO}_{2}$ nanocylinders of radius $R=180 \mathrm{~nm}$ with period $d=380 \mathrm{~nm}$. The inset shows the region around $k=0$ ( $\Gamma$ point) where third and fourth bands are degenerate, exhibiting the upper band isotropy over a sufficiently wide $k$-range.

The plausible behavior of our all-dielectric array as DZM about the doubly degenerate point now needs to be verified. Therefore, we show the transmission of a plane wave through an infinite slab for an increasing angle of incidence in Figure 4. A remarkable DZM behavior is observed, with a total transmission only very near normal incidence (a constant phase front) and an abrupt total reflection beyond a narrow admittance angle $\sim 3^{\circ}$. Such total reflection is preserved up to a large angle of incidence $\geq 30^{\circ}$, which corresponds to the wavevector region shown above (see Figure 3), where the two photonic bands bend and cross each other along the $\Gamma M$ direction. 


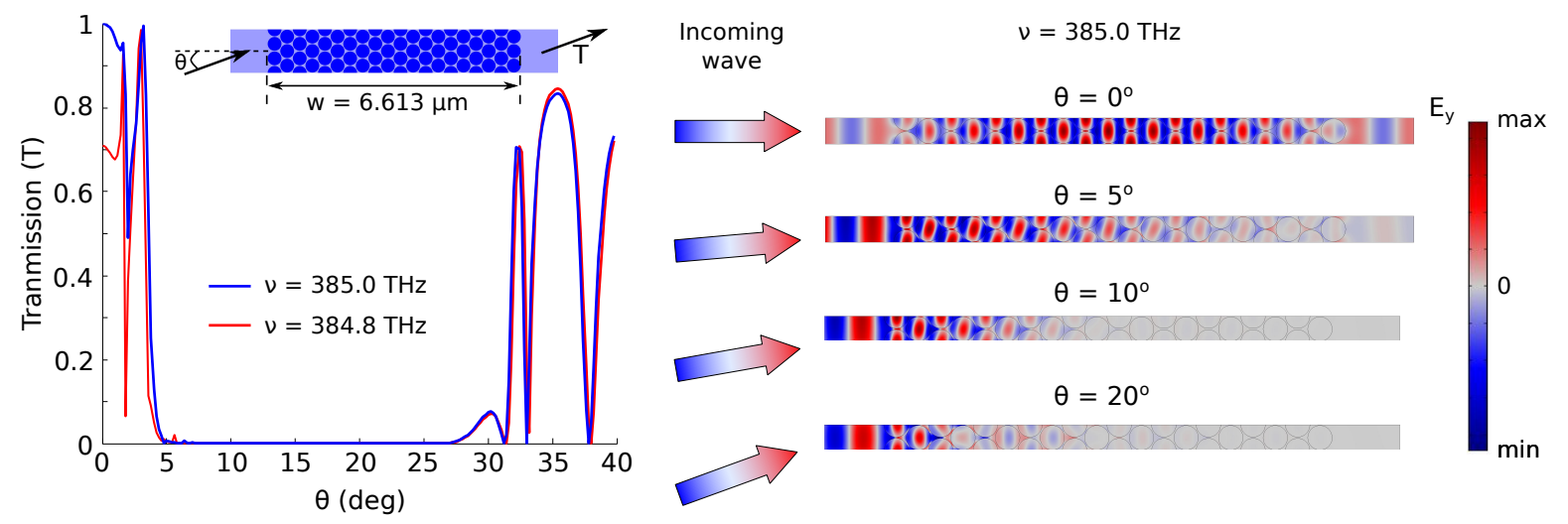

Figure 4. Left: Transmission as a function of the angle of incidence $\theta$ of a TE-polarized plane wave through an infinite slab of width $W=6.613 \mu \mathrm{m}$ made of an array of $\mathrm{TiO}_{2}$ nanocylinders of radius $R=180 \mathrm{~nm}$ with period $d=380 \mathrm{~nm}$ for two frequencies at/near the doubly degenerate point. The inset depicts the configuration. Right: Electric field maps across the slabs for various relevant angles of incidence at fixed frequency $v=385 \mathrm{THz}$.

Finally, two devices are numerically simulated where the DZM behavior is evidenced. First, we show in Figure 5 a finite thin slab similar to the infinite one considered in Figure 4. The zero-phase-delay across the slab can be observed, although a finer structure of the electric field within the rods is also appreciable. The wave front is fully transmitted with negligible absorption $\left(n^{\text {eff }} \sim 0\right)$ and a nearly negligible reflection at the entrance face. Moreover, a prism acting as a beam splitter is also shown in Figure 5, where some disturbances in the input face can be observed due to unwanted reflection, and in the output corner. Corresponding videos are included as supplementary information. The phase front evolution can be clearly observed, evidencing this quasi-constant phase (quasi-infinite phase velocity) across both devices. Particularly, in the case of the prism, it can be observed that the wavefront actually follows the inclination of the output faces, leading to two outgoing beams (except for some spurious diffraction near the prism edge) emerging from these two faces of the prism, as is observed in beam splitters.
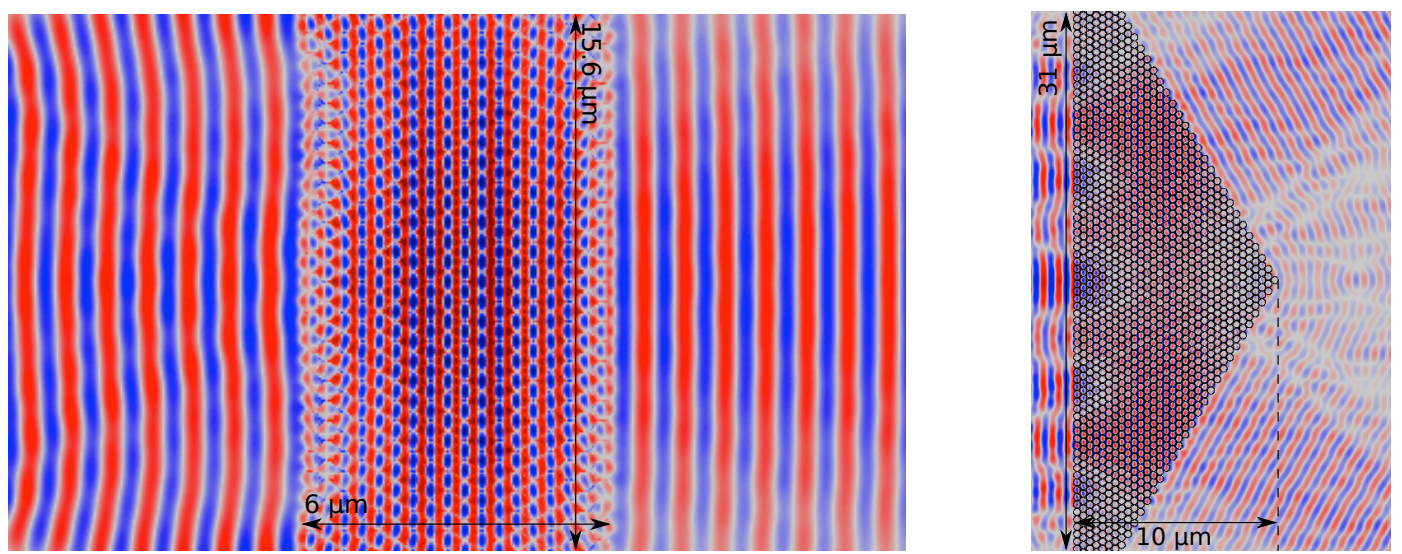

Figure 5. Transverse electric field snapshots for two finite arrangements of an array of $\mathrm{TiO}_{2}$ nanocylinders with a radius $R=180 \mathrm{~nm}$ and a period $d=380 \mathrm{~nm}$, on which a TE-polarized Gaussian beam impinges at the frequency of the doubly degenerate point described above: a slab (left) and a prism (beam splitter, right) on which a Gaussian beam impinges, revealing the resulting DZM behavior of the nanocylinder array. Corresponding videos are included in the Supplementary Information where the phase front evolution is clearly observed. 


\section{Conclusions}

We have theoretically explored a means of obtaining an impedance-matched, double-zero optical metamaterial on the basis of weakly (electric/magnetic) resonant meta-atoms, exploiting their off-resonant, negative polarizibilities on the blue side of the overlapping resonances. Metal-oxide nanowires are proposed as good candidates to fulfill such conditions, revealing through effective medium theory that this is the case for $\mathrm{TiO}_{2}$ cylinders in the optical domain. Numerical analysis of the photonic band structure in TE polarization for a closely packed triangular lattice of $\mathrm{TiO}_{2}$ rods reveals a doubly degenerate point at $k=0$ at the third and fourth bands. The transmission of plane waves at frequencies close to the degeneracy exhibit a DZM behavior with a transmission only at near normal incidence and a total reflection for an increasing angle up to $\sim 30^{\circ}$, beyond which the two bands bend and cross each other. Further numerical simulations for configurations such as a finite slab and a beam splitter (prism) neatly manifest remarkable DZM-expected phenomena, which have fascinating applications in optics and engineering. Our results aid in the actualization of impedance-matched, low-loss (bulky) DZMs (in the visible and throughout the EM spectrum) with moderately large index dielectrics, which might ease optical DZM fabrication through state-of-the-art metal-oxide nanowire arrays [60,61].

Supplementary Materials: The following are available online at http:/ www.mdpi.com/2304-6732/5/2/7/s1, Video S1: Transverse electric field in a full harmonic cycle for a finite slab made of $\mathrm{TiO}_{2}$-cylinder triangular array shown in Figure 5. Video S2: Transverse electric field in a full harmonic cycle for a prism (beam splitter) made of $\mathrm{TiO}_{2}$-cylinder triangular array shown in Figure 5.

Acknowledgments: This work was supported in part by the Spanish Ministerio de Economía, Industria y Competitividad (projects Consolider-Ingenio EMET CSD2008-000666 and LENSBEAM FIS2015-69295-C3-2-P). We acknowledge helpful discussions with L. S. Froufe-Pérez, J. J. Sáenz, R. Marqués, and M. Nieto-Vesperinas.

Author Contributions: The original idea of the article was conceived by all the authors. D.R.A. and R.P.-D. performed the analytical and numerical calculation. J.S.S.-G wrote the paper.

Conflicts of Interest: The authors declare no conflict of interest.

\section{Abbreviations}

The following abbreviations are used in this manuscript:

$\begin{array}{ll}\text { ENZ } & \varepsilon \text {-near-zero } \\ \text { DZM } & \text { Double-zero metamaterials } \\ \text { TE } & \text { Transverse electric } \\ \text { TM } & \text { Transverse magnetic } \\ \text { EM } & \text { Electromagnetic }\end{array}$

\section{References}

1. Smith, D.; Padilla, W.; Vier, D.; Nemat-Nasser, S.; Schultz, S. Composite Medium with Simultaneously Negative Permeability and Permittivity. Phys. Rev. Lett. 2000, 84, 4184-4187.

2. Pendry, J.B. Negative refraction makes a perfect lens. Phys. Rev. Lett. 2000, 85, 3966-3969.

3. Engheta, N.; Ziolkowski, R. Metamaterials, Physics and Engineering Explorations; IEEE-Wiley: New York, NY, USA, 2006.

4. Marques, R.; Martin, F.; Sorolla, M. Metamaterials with Negative Parameters: Theory, Design and Microwave Applications; Wiley: New York, NY, USA, 2007.

5. Enoch, S.; Tayeb, G.; Sabouroux, P.; Guérin, N.; Vincent, P. A Metamaterial for Directive Emission. Phys. Rev. Lett. 2002, 89, 213902.

6. Ziolkowski, R. Propagation in and scattering from a matched metamaterial having a zero index of refraction. Phys. Rev. E 2004, 70, 046608.

7. Engheta, N. Pursuing Near-Zero Response. Science 2013, 340, 286-287.

8. Liberal, I.; Engheta, N. The rise of near-zero-index technologies. Science 2017, 358, 1540-1541.

9. Liberal, I.; Engheta, N. Near-zero refractive index photonics. Nat. Photonics 2017, 11, 149-158. 
10. Silveirinha, M.; Engheta, N. Tunneling of Electromagnetic Energy through Subwavelength Channels and Bends using $\epsilon$-Near-Zero Materials. Phys. Rev. Lett. 2006, 97, 157403.

11. Silveirinha, M.; Engheta, N. Theory of supercoupling, squeezing wave energy, and field confinement in narrow channels and tight bends using $\epsilon$ near-zero metamaterials. Phys. Rev. B 2007, 76, 245109.

12. Alù, A.; Silveirinha, M.; Salandrino, A.; Engheta, N. Epsilon-near-zero metamaterials and electromagnetic sources: Tailoring the radiation phase pattern. Phys. Rev. B 2007, 75, 155410.

13. Silveirinha, M.; Engheta, N. Design of matched zero-index metamaterials using nonmagnetic inclusions in $\epsilon$-near-zero media. Phys. Rev. B 2007, 75, 075119.

14. Edwards, B.; Alù, A.; Young, M.; Silveirinha, M.; Engheta, N. Experimental Verification of Epsilon-Near-Zero Metamaterial Coupling and Energy Squeezing Using a Microwave Waveguide. Phys. Rev. Lett. 2008, 100, 033903.

15. Huang, X.; Lai, Y.; Hang, Z.H.; Zheng, H.; Chan, C.T. Dirac cones induced by accidental degeneracy in photonic crystals and zero-refractive-index materials. Nat. Mater. 2011, 10, 582-586.

16. Feng, S. Loss-Induced Omnidirectional Bending to the Normal in $\epsilon$-Near-Zero Metamaterials. Phys. Rev. Lett. 2012, 108, 193904.

17. Vesseur, E.; Coenen, T.; Caglayan, H.; Engheta, N.; Polman, A. Experimental Verification of $n=0$ Structures for Visible Light. Phys. Rev. Lett. 2013, 110, 013902.

18. Alù, A.; Engheta, N. All Optical Metamaterial Circuit Board at the Nanoscale. Phys. Rev. Lett. 2009, 103, 143902.

19. Alu, A.; Engheta, N. Coaxial-to-Waveguide Matching With Epsilon-Near-Zero Ultranarrow Channels and Bends. IEEE Trans. Antennas Propag. 2010, 58, 328-339.

20. Yang, J.J.; Francescato, Y.; Maier, S.A.; Mao, F.; Huang, M. Mu and epsilon near zero metamaterials for perfect coherence and new antenna designs. Opt. Express 2014, 22, 9107-9114.

21. Alù, A.; Engheta, N. Boosting Molecular Fluorescence with a Plasmonic Nanolauncher. Phys. Rev. Lett. 2009, 103, 043902.

22. Suchowski, H.; O’Brien, K.; Wong, Z.J.; Salandrino, A.; Yin, X.; Zhang, X. Phase mismatch-free nonlinear propagation in optical zero-index materials. Science 2013, 342, 1223-1226.

23. Caspani, L.; Kaipurath, R.P.M.; Clerici, M.; Ferrera, M.; Roger, T.; Kim, J.; Kinsey, N.; Pietrzyk, M.; Di Falco, A.; Shalaev, V.M.; et al. Enhanced Nonlinear Refractive Index in $\epsilon$-Near-Zero Materials. Phys. Rev. Lett. 2016, 116, 233901.

24. Liberal, I.; Mahmoud, A.M.; Li, Y.; Edwards, B.; Engheta, N. Photonic doping of epsilon-near-zero media. Science 2017, 355, 1058-1062.

25. Rodríguez-Fortuño, F.J.; Vakil, A.; Engheta, N. Electric Levitation Using epsilon-Near-Zero Metamaterials. Phys. Rev. Lett. 2014, 112, 033902.

26. Jeong-Hae, L. Dual Property of Mu-near zero to Epsilon-near Zero Material. J. Korean Phys. Soc. 2010, 57, 51.

27. Marcos, J.S.; Silveirinha, M.G.; Engheta, N. $\mu$-near-zero supercoupling. Phys. Rev. B 2015, 91, 195112.

28. Torres, V.; Pacheco-Peña, V.; Rodríguez-Ulibarri, P.; Navarro-Cía, M.; Beruete, M.; Sorolla, M.; Engheta, N. Terahertz epsilon-near-zero graded-index lens. Opt. Express 2013, 21, 9156-9166.

29. Li, Y.; Kita, S.; Muñoz, P.; Reshef, O.; Vulis, D.I.; Yin, M.; Lončar, M.; Mazur, E. On-chip zero-index metamaterials. Nat. Photonics 2015, 9, 738-742.

30. Moitra, P.; Yang, Y.; Anderson, Z.; Kravchenko, I.I.; Briggs, D.P.; Valentine, J. Realization of an all-dielectric zero-index optical metamaterial. Nat. Photonics 2013, 7, 791-795.

31. Maas, R.; Parsons, J.; Engheta, N.; Polman, A. Experimental realization of an epsilon-near-zero metamaterial at visible wavelengths. Nat. Photonics 2013, 7, 907-912.

32. Pollard, R.J.; Murphy, A.; Hendren, W.R.; Evans, P.R.; Atkinson, R.; Wurtz, G.A.; Zayats, A.V.; Podolskiy, V.A. Optical Nonlocalities and Additional Waves in Epsilon-Near-Zero Metamaterials. Phys. Rev. Lett. 2009, 102, 127405.

33. Soukoulis, C.M.; Wegener, M. Optical metamaterials-More bulky and less lossy. Science 2010, 330, $1633-1634$.

34. Paniagua-Domínguez, R.; Abujetas, D.R.; Sánchez-Gil, J.A. Ultra low-loss, isotropic optical negative-index metamaterial based on hybrid metal-semiconductor nanowires. Sci. Rep. 2013, 3, 1507.

35. Abujetas, D.R.; Paniagua-Domínguez, R.; Nieto-Vesperinas, M.; Sánchez-Gil, J.A. Photonic band structure and effective medium properties of doubly-resonant core-shell metallo-dielectric nanowire arrays: Low-loss, isotropic optical negative-index behavior. J. Opt. 2015, 17, 125104.

36. Mahmoud, A.M.; Engheta, N. Wave-matter interactions in epsilon-and-mu-near-zero structures. Nat. Commun. 2014, 5, 1-7. 
37. Wheeler, M.; Aitchison, J.; Mojahedi, M. Three-dimensional array of dielectric spheres with an isotropic negative permeability at infrared frequencies. Phys. Rev. B 2005, 72, 193103.

38. Schuller, J.A.; Zia, R.; Taubner, T.; Brongersma, M.L. Dielectric Metamaterials Based on Electric and Magnetic Resonances of Silicon Carbide Particles. Phys. Rev. Lett. 2007, 99, 107401.

39. Zhao, Q.; Kang, L.; Du, B.; Zhao, H.; Xie, Q.; Huang, X.; Li, B.; Zhou, J.; Li, L. Experimental Demonstration of Isotropic Negative Permeability in a Three-Dimensional Dielectric Composite. Phys. Rev. Lett. 2008, 101, 027402.

40. Popa, B.I.; Cummer, S. Compact Dielectric Particles as a Building Block for Low-Loss Magnetic Metamaterials. Phys. Rev. Lett. 2008, 100, 207401.

41. Vynck, K.; Felbacq, D.; Centeno, E.; Căbuz, A.; Cassagne, D.; Guizal, B. All-Dielectric Rod-Type Metamaterials at Optical Frequencies. Phys. Rev. Lett. 2009, 102, 133901.

42. Zhao, Q.; Zhou, J.; Zhang, F.; Lippens, D. Mie resonance-based dielectric metamaterials. Mater. Today 2009, 12, 60-69.

43. Evlyukhin, A.B.; Reinhardt, C.; Seidel, A.; Luk'yanchuk, B.S.; Chichkov, B.N. Optical response features of Si-nanoparticle arrays. Phys. Rev. B 2010, 82, 045404.

44. Jelinek, L.; Marqués, R. Artificial magnetism and left-handed media from dielectric rings and rods. J. Phys. Condens. Matter 2010, 22, 025902.

45. García-Etxarri, A.; Gómez-Medina, R.; Froufe-Pérez, L.S.; López, C.; Chantada, L.; Scheffold, F.; Aizpurua, J.; Nieto-Vesperinas, M.; Sáenz, J.J. Strong magnetic response of submicron Silicon particles in the infrared. Opt. Express 2011, 19, 4815-4826.

46. Evlyukhin, A.B.; Novikov, S.M.; Zywietz, U.; Eriksen, R.L.; Reinhardt, C.; Bozhevolnyi, S.I.; Chichkov, B.N. Demonstration of Magnetic Dipole Resonances of Dielectric Nanospheres in the Visible Region. Nano Lett. 2012, 12, 3749-3755.

47. Kuznetsov, A.I.; Miroshnichenko, A.E.; Fu, Y.H.; Zhang, J.; Luk'yanchuk, B. Magnetic light. Sci. Rep. $2012,2,492$.

48. Paniagua-Domínguez, R.; Froufe-Pérez, L.S.; Sáenz, J.J.; Sánchez-Gil, J.A. Localized magnetic plasmons in all-dielectric $\mu<0$ metastructures. Phys. Rev. B 2015, 91, 235120.

49. Kuznetsov, A.I.; Miroshnichenko, A.E.; Brongersma, M.L.; Kivshar, Y.S.; Luk'yanchuk, B. Optically resonant dielectric nanostructures. Science 2016, 354, 846.

50. Kruk, S.S.; Camacho-Morales, R.; Xu, L.; Rahmani, M.; Smirnova, D.A.; Wang, L.; Tan, H.H.; Jagadish, C.; Neshev, D.N.; Kivshar, Y.S. Nonlinear Optical Magnetism Revealed by Second-Harmonic Generation in Nanoantennas. Nano Lett. 2017, 17, 3914-3918.

51. Yang, Y.; Miroshnichenko, A.E.; Kostinski, S.V.; Odit, M.; Kapitanova, P.; Qiu, M.; Kivshar, Y.S. Multimode directionality in all-dielectric metasurfaces. Phys. Rev. B 2017, 95, 165426.

52. Paniagua-Domínguez, R.; López-Tejeira, F.; Marqués, R.; Sánchez-Gil, J.A. Metallo-dielectric core-shell nanospheres as building blocks for optical three-dimensional isotropic negative-index metamaterials. New J. Phys. 2011, 13, 123017.

53. Morits, D.; Simovski, C. Isotropic negative refractive index at near infrared. J. Opt. 2012, 14, 125102.

54. Mirzaei, A.; Shadrivov, I.V.; Miroshnichenko, A.E.; Kivshar, Y.S. Cloaking and enhanced scattering of core-shell plasmonic nanowires. Opt. Express 2013, 21, 10454-10459.

55. Monticone, F.; Alù, A. Do Cloaked Objects Really Scatter Less? Phys. Rev. X 2013, 3, 041005.

56. Paniagua-Domínguez, R.; Abujetas, D.R.; Froufe-Pérez, L.S.; Sáenz, J.J.; Sánchez-Gil, J.A. Broadband telecom transparency of semiconductor-coated metal nanowires: More transparent than glass. Opt. Express 2013, 21, 22076-22089.

57. Liu, W.; Miroshnichenko, A.E.; Oulton, R.F.; Neshev, D.N.; Hess, O.; Kivshar, Y.S. Scattering of core-shell nanowires with the interference of electric and magnetic resonances. Opt. Lett. 2013, 38, 2621-2624.

58. Wu, Y.; Li, J.; Zhang, Z.Q.; Chan, C. Effective medium theory for magnetodielectric composites: Beyond the long-wavelength limit. Phys. Rev. B 2006, 74, 085111.

59. Palik, E.D. Handbook of Optical Constants of Solids; Academic Press: Cambridge, MA, USA, 1998; Volume 3, p. 900.

60. Dai, L.; Sow, C.H.; Lim, C.T.; Tan, V.B.C. Metal Oxide Nanowires-Structural and Mechanical Properties. In Nanowires_Fundamental Research; Hashim, A., Ed.; InTech: Singapore, 2011; Chapter 9.

61. Wu, J.M.; Shih, H.C.; Wu, W.T. Formation and photoluminescence of single-crystalline rutile $\mathrm{TiO}_{2}$ nanowires synthesized by thermal evaporation. Nanotechnology 2006, 17, 105-109. 
62. Li, Y.; Wu, Y.; Chen, X.; Mei, J. Selection rule for Dirac-like points in two-dimensional dielectric photonic crystals. Opt. Express 2013, 21, 7699-7711.

63. Marini, A.; García de Abajo, F.J. Self-organization of frozen light in near-zero-index media with cubic nonlinearity. Sci. Rep. 2016, 6, 20088.

(C) 2018 by the authors. Licensee MDPI, Basel, Switzerland. This article is an open access article distributed under the terms and conditions of the Creative Commons Attribution (CC BY) license (http:/ / creativecommons.org/licenses/by/4.0/). 